\title{
Competing reaction processes on a lattice as a paradigm for catalyst deactivation
}

\author{
E. Abad ${ }^{1, *}$ and J. J. Kozak ${ }^{2,3}$ \\ ${ }^{1}$ Departamento de Física Aplicada and Instituto de Computación Científica Avanzada (ICCAEX), Centro Universitario de Mérida, \\ Universidad de Extremadura, E-06800 Mérida, Spain \\ ${ }^{2}$ DePaul University, 243 South Wabash, Chicago, Illinois 60604-2301, USA \\ ${ }^{3}$ Beckman Institute, Caltech, Pasadena, California 91125, USA
}

(Received 31 July 2014; published 6 February 2015)

\begin{abstract}
We mobilize both a generating function approach and the theory of finite Markov processes to compute the probability of irreversible absorption of a randomly diffusing species on a lattice with competing reaction centers. We consider an $N$-site lattice populated by a single deep trap, and $N-1$ partially absorbing traps (absorption probability $0<s<1$ ). The influence of competing reaction centers on the probability of reaction at a target site (the deep trap) and the mean walk length of the random walker before localization (a measure of the reaction efficiency) are computed for different geometries. Both analytic expressions and numerical results are given for reactive processes on two-dimensional surfaces of Euler characteristic $\Omega=0$ and $\Omega=2$. The results obtained allow a characterization of catalyst deactivation processes on planar surfaces and on catalyst pellets where only a single catalytic site remains fully active (deep trap), the other sites being only partially active as a result of surface poisoning. The central result of our study is that the predicted dependence of the reaction efficiency on system size $N$ and on $s$ is in qualitative accord with previously reported experimental results, notably catalysts exhibiting selective poisoning due to surface sites that have different affinities for chemisorption of the poisoning agent (e.g., acid zeolite catalysts). Deviations from the efficiency of a catalyst with identical sites are quantified, and we find that such deviations display a significant dependence on the topological details of the surface (for fixed values of $N$ and $s$ we find markedly different results for, say, a planar surface and for the polyhedral surface of a catalyst pellet). Our results highlight the importance of surface topology for the efficiency of catalytic conversion processes on inhomogeneous substrates, and in particular for those aimed at industrial applications. From our exact analysis we extract results for the two limiting cases $s \approx 1$ and $s \approx 0$, corresponding respectively to weak and strong catalyst poisoning (decreasing $s$ leads to a monotonic decrease in the efficiency of catalytic conversion). The results for the $s \approx 0$ case are relevant for the dual problem of light-energy conversion via trapping of excitations in the chlorophyll antenna network. Here, decreasing the probability of excitation trapping $s$ at sites other than the target molecule does not result in a decrease of the efficiency as in the catalyst case, but rather in enhanced efficiency of light-energy conversion, which we characterize in terms of $N$ and $s$. The one-dimensional case and its connection with a modified version of the gambler's ruin problem are discussed. Finally, generalizations of our model are described briefly.
\end{abstract}

DOI: 10.1103/PhysRevE.91.022106

PACS number(s): 05.40.Fb, 05.50.+q, 68.43.Mn

\section{INTRODUCTION}

Experimental observations in surface catalysis have been analyzed using a variety of continuum and lattice models [1]. Aspects of catalytic behavior have been explored using classical chemical kinetics [2,3] and diffusion-reaction theory. Studies based on a (Fickian) second-order partial differential equation with a linear dependence on the concentration(s) account for behavior in the near-equilibrium regime. Far-fromequilibrium phenomena (rate oscillations, spatial-temporal patterns and chaos) have been modelled by considering a nonlinear dependence on the relevant concentration(s) [4]. As for lattice models, the main focus has been on simulations, principally Monte Carlo studies on planar lattices. Effects of catalytic poisoning for specific reactions have been explored by designing algorithms, some general enough to account for "feedback" between surface concentration and absorption rate [5-7].

In this study, we examine the role of competing reaction centers in influencing the overall efficiency of a diffusion-

\footnotetext{
*Author to whom correspondence should be addressed: eabad@unex.es
}

reaction process. In the problem of catalyst degradation, deactivation of reaction centers leads to catalyst poisoning, usually an unwanted effect (although in some systems it can enhance the selectivity of the reaction). By contrast, in the dual problem of photosynthesis, deactivation of all competing reaction centers in the chlorophyll antenna network except for the target molecule optimizes light-energy conversion.

The present contribution departs from earlier studies based on a lattice model in two important respects. First, in our lattice-statistical approach we place emphasis on obtaining analytic expressions to display the dependence of catalyst deactivation on such factors as domain size and extent of surface degradation. And second, we consider explicitly the geometry of the template. The surface of a planar lattice subject to periodic boundary conditions is characterized topologically by Euler characteristic $\Omega=0$, whereas the surface of a catalyst pellet by $\Omega=2$. Empirically, surface deactivation and turnover on these two templates (both of Euclidean dimension $d=2$, and a domain specified by the number $N$ of surface sites and their connectivity $\gamma$ ) are known to be different, and this we will quantify using the lattice-statistical approach introduced here, as will now be described. 
For a freshly prepared catalyst, with no sites "poisoned," a reactant hitting the surface can react at any site. In a lattice model, this situation would correspond to all sites being "deep traps." Considering now the effect of poisoning by some agent, one can distinguish two situations, viz., uniform degradation of the catalytic activity of all sites (which then play the role of identical imperfect traps) vs nonuniform site degradation. In the latter case, the simplest setting corresponds to the case where one site (the deep trap) remains totally active, but the $N-1$ satellite sites are uniformly degraded, so that only with a certain probability $s$, catalytic conversion takes place at these satellite sites. Values of the probability $s \leqslant 1$ characterize deactivation of the surface until in the limit $s=0$ there remains only one fully active site. Such inhomogeneous poisoning of lattice sites is one of the common pathways leading to so-called selective poisoning, a well studied phenomenon resulting in nonlinear behavior of the catalytic activity as a function of poison concentration [1]. A fundamental question for current research in surface chemistry amounts to elucidating the role of substrate geometry.

Catalyst poisoning can have two effects. First, the number of catalytic sites (out of $N$ sites in the domain) that can promote efficient conversion decreases; and second, the average distance that a reactant must diffuse before undergoing reaction (in lattice models, the so-called mean walk length $\langle n\rangle$ ) necessarily increases. It is in calculating this average distance, $\langle n\rangle$, that differences in catalyst deactivation for planar surfaces versus closed polyhedral surfaces mimicking catalyst pellets can be quantified.

In this study, we mobilize both the method of generating functions and the theory of finite Markov processes to study catalyst deactivation. With respect to the latter approach, this study draws on two earlier studies [8,9], where a method based on the theory of finite Markov processes was presented to calculate numerically exact values of the expected walk length $\langle n\rangle$ of a random walker performing a Pólya walk on a regular $d$-dimensional $N$-site lattice with a single perfect trap (absorption probability equal to 1 as soon as the walker steps on the trapping site) and $N-1$ imperfect traps (absorption probability equal to $s$ when the walker steps on any other site). The sitespecific walk lengths of the walker from any/all sites of the host lattice are determined from the inverse of the fundamental matrix of Markov chain theory. This approach can be mobilized for any lattice (translational invariance is not a constraint), and hence can be applied to template topologies of either Euler characteristic $\Omega$. While, as a general rule, the Markovian approach does not lend itself easily to obtaining analytic results, its application leads straightforwardly to results which are numerically exact and satisfy key invariance relations.

We wish to take advantage of insights gained using both of the above strategies. By making use of the generating function formalism for random walks developed by Montroll and Weiss [10], we rederive and significantly extend some results obtained previously [9], thus establishing the relationship between the generating-function approach and Markov theory for lattices with competing reaction centers. The generating function approach is only easily applicable to translationinvariant lattices, and in this sense is less flexible than the Markov method. However, the approach has the significant advantage of providing a straightforward way of calculating higher order moments of the walk length (the moments are usually expressible in terms of suitable derivatives of generating functions). Using this method, it is usually simpler to find explicit, closed expressions for the moments in terms of lattice size $N$, rather than having to develop analytic solutions from numerical data generated for lattices of increasing size $N$ using the Markov approach.

In light of recent work by Yuste, Abad, and Lindenberg [11], we draw the reader's attention to the fact that homogeneous absorption at each of the imperfect traps may be interpreted as a special case of a recently developed generating-function theory for so-called "mortal walkers," i.e., walkers that have a finite probability of "dying" at each time step [11]. For purposes of the present study, that probability will be assumed to be stationary and homogeneous, although these restrictions can be relaxed in future work. The approach developed in Refs. [11] assumes translational invariance of the host lattice; in our lattice model this condition is implemented by imposing periodic boundary conditions on a planar template, and corresponds topologically to mapping the planar lattice onto the surface of a torus. The significant advantage gained in mobilizing the theory of generating functions is that analytic expressions can be obtained for the mean walk length (see Sec. II).

Placing the present study in a larger context, to the best of our knowledge the problem of random walks with competing decay channels seems to have received surprisingly little attention in the literature. Rosenstock was one of the first (if not the first) to address this problem, as he considered a walker subject to spontaneous emission moving on a lattice where a fraction of the sites are perfect traps [12]. He computed for various cases the probability that the walker dies by spontaneous emission rather than by stepping on a trap. Various authors subsequently elaborated further versions of this problem in different dimensions [13-15]. Several years later, Den Hollander and Kasteleyn considered a similar problem, a lattice with a periodic array of partially absorbing traps [16]. Evans and Nord reviewed the connection between the generating function approach and the Markov method for multiple trap problems [17].

Finally, in a study of particular relevance to the problem studied here, Weiss [18] dealt with a random walk on a lattice with partial traps, each having different absorption probabilities. An expression was given for the probability of the walker being absorbed at a given trap in terms of generating functions. The examples given in that paper focused mainly on the case of one or two partially absorbing traps.

In what follows, we consider the case where all but one of the lattice sites have the same absorption probability. Thus, the walker is "mortal," i.e., it has a constant probability of "dying" per unit step as long as it does not step on the deep trap. In related applications, for example those describing spontaneous light emission or radioactive decay, background absorption can be regarded not as a property of the substrate but rather as a stochastic death process due to the intrinsic properties of the diffusing particle. However, we shall hereafter mainly focus on the problem of nonuniform catalyst degradation, where the absorption probability of each site is interpreted to be directly related to the (local) catalytic activity of the substrate.

The plan of the paper is as follows. In Sec. II we derive explicit formulas for the probability of the walker being 
absorbed at the deep trap and for the walk length for specific initial configurations of the walker and the deep trap (these two quantities are intimately related). We also give a general expression for the walk length $\langle n\rangle$ when the average over all possible configurations is taken. In Sec. III we briefly discuss the one-dimensional case and the connection with the so-called ruin problem. In Sec. IV we focus on the specific case of a $d=2$ dimensional lattice subject to periodic boundary conditions and show how to recover and then generalize the results in Sec. V of Ref. [9]. In Sec. V we summarize the numerical results obtained for both catalyst surfaces and catalyst pellets, and compare them to results for the case where two deep traps are placed on a lattice with no background absorption. Finally, in Sec. VI we discuss the relevance of our analytical and numerical results to the experimental problem of catalyst deactivation.

\section{GENERAL FRAMEWORK}

We consider a random walker and an immobile deep trap placed at two different sites on an $N$-site, $d$-dimensional periodic lattice. The site at which the trap is placed is fully absorbing, whereas all other sites (including the one from which the random walker starts off, that is, the origin $\overrightarrow{0}$ ) are partially absorbing, with absorption probability $s$. Thus, two competing absorption mechanisms (one localized at the deep trap and the other distributed over the whole lattice) are at play. In the present problem, the deep trap represents a catalytic site that remains fully active, whereas the surrounding sites of the substrate are partially poisoned.

\section{A. Quantities for specific walker-trap configurations}

We first ask, what is the trajectory-averaged walk length $\langle n\rangle_{\vec{\ell}}$ for a specific initial location of the trap with respect to the random walker. The position of the trap will be specified by the $d$-dimensional vector $\vec{\ell}=\left(\ell_{1}, \ell_{2}, \ldots, \ell_{d}\right) \neq \overrightarrow{0}$. Following the convention used in Refs. [8,9], we take $\langle n\rangle_{\vec{\ell}} \equiv 1$ if the walker is trapped at the very outset. The $k$ th order moment of the walk length is

$$
\left\langle n^{k}\right\rangle_{\vec{\ell}}=\sum_{n=1}^{\infty} n^{k} A_{n}(\vec{\ell})
$$

where $A_{n}(\vec{\ell})$ is the probability that the walker is absorbed after exactly $n$ steps, which is the sum of the probabilities for two mutually exclusive events, viz., that the walker is absorbed at the deep trap or that it is absorbed at any other site.

Let $F_{n}(\vec{\ell})$ denote the probability that the walker reaches the deep trap exactly at the $n$th step conditional on its not having been absorbed previously at any other site [thus, $F_{n}(\vec{\ell})$ plays the role of a conditional first-passage probability]. One then has

$$
A_{n}(\vec{\ell})=(1-s)^{n} F_{n}(\vec{\ell})+(1-s)^{n-1} s U_{n-1}(\vec{\ell}),
$$

where $U_{n}(\vec{\ell}) \equiv 1-\sum_{j=0}^{n} F_{j}(\vec{\ell})$ is the survival probability of the walker after $n$ steps when sites other than the deep trap are nonabsorbing $(s=0)$. The terms on the right-hand side of the above equation have the following interpretation: the term $(1-s)^{n} F_{n}(\vec{\ell})$ is the probability that the walker is not absorbed at any of the partially absorbing sites before the $n$th jump and that this jump results in stepping on the deep trap. On the other hand, the second term states that, in order to be absorbed at a partial trap after exactly $n$ steps, the walker must have survived both absorption at the deep trap and at the partially absorbing traps during the previous $n-1$ steps. The probability for this to happen is $(1-s)^{n-1} U_{n-1}(\vec{\ell})$.

Hence, we have

$$
\left\langle n^{k}\right\rangle_{\vec{\ell}}=\sum_{n=1}^{\infty} n^{k}\left\{(1-s)^{n} F_{n}(\vec{\ell})+(1-s)^{n-1} s U_{n-1}(\vec{\ell})\right\} .
$$

In particular, for $k=0$ Eq. (1) yields the zeroth order moment:

$$
\left\langle n^{0}\right\rangle_{\vec{\ell}}=A_{T}(\vec{\ell})+A_{O}(\vec{\ell}) \equiv 1,
$$

where $A_{T}(\vec{\ell}) \equiv \sum_{n=1}^{\infty}(1-s)^{n} F_{n}(\vec{\ell})$ is the probability that the walker terminates its walk at the deep trap, and $A_{O}(\vec{\ell}) \equiv$ $\sum_{n=1}^{\infty}(1-s)^{n-1} s U_{n-1}(\vec{\ell})$ is the probability that it is absorbed at any other site. These probabilities are most conveniently expressed in terms of generating functions. Introducing the generating function $F(\vec{\ell}, z)=\sum_{n=0}^{\infty} F_{n}(\vec{\ell}) z^{n}$ there results

$$
\begin{aligned}
A_{T}(\vec{\ell}) & \left.\equiv F(\vec{\ell}, z)\right|_{z=1-s}-F_{0}(\vec{\ell})=\left.F(\vec{\ell}, z)\right|_{z=1-s}-0 \\
& =F(\vec{\ell}, 1-s)=\frac{P(\vec{\ell}, 1-s)}{P(\overrightarrow{0}, 1-s)} .
\end{aligned}
$$

In the last line we have made use of the fact that $F(\vec{\ell}, z) \equiv P(\vec{\ell}, z) / P(\overrightarrow{0}, z)$, where $P(\vec{\ell}, z)=\sum_{n=0}^{\infty} P_{n}(\vec{\ell}) z^{n}$ is the generating function for the probability $P_{n}(\vec{\ell})$ of the walker being at the lattice site $\vec{\ell}$ after exactly $n$ steps in an unrestricted random walk $[10(\mathrm{~b})]$.

On the other hand, $A_{O}(\vec{\ell})$ can be written as $A_{O}(\vec{\ell}) \equiv$ $s U(\vec{\ell}, 1-s)$, where the generating function $U(\vec{\ell}, z)=$ $\sum_{n=0}^{\infty} U_{n}(\vec{\ell}) z^{n}$ has been introduced. The specific expression of $U(\vec{\ell}, z)$ in terms of $F(\vec{\ell}, z) \equiv P(\vec{\ell}, z) / P(\overrightarrow{0}, z)$ is available (see, for example, Ref. [19], p. 158):

$$
U(\vec{\ell}, z)=\frac{1}{1-z}\left[1-\frac{P(\vec{\ell}, z)}{P(\overrightarrow{0}, z)}\right]=\frac{1}{1-z}[1-F(\vec{\ell}, z)] .
$$

Hence, $A_{O}(\vec{\ell}) \equiv 1-F(\vec{\ell}, 1-s)$, as required by the normalization condition for the splitting probabilities.

We next turn to the equation for the first order moment of the walk length distribution, that is,

$$
\langle n\rangle_{\vec{\ell}}=\sum_{n=1}^{\infty} n\left\{(1-s)^{n} F_{n}(\vec{\ell})+(1-s)^{n-1} s U_{n-1}(\vec{\ell})\right\} .
$$

Equation (5) can be rewritten as follows:

$$
\begin{aligned}
\langle n\rangle_{\vec{\ell}}= & (1-s) \sum_{n=1}^{\infty} n F_{n}(\vec{\ell})(1-s)^{n-1} \\
& +s \sum_{n=1}^{\infty}(n-1) U_{n-1}(\vec{\ell})(1-s)^{n-1} \\
& +s \sum_{n=1}^{\infty} U_{n-1}(\vec{\ell})(1-s)^{n-1}
\end{aligned}
$$


or, in terms of generating functions,

$$
\begin{aligned}
\langle n\rangle_{\vec{\ell}}= & \left.(1-s) \frac{\partial}{\partial z} F(\vec{\ell}, z)\right|_{z=1-s}+\left.s(1-s) \frac{\partial}{\partial z} U(\vec{\ell}, z)\right|_{z=1-s} \\
& +\left.s U(\vec{\ell}, z)\right|_{z=1-s} .
\end{aligned}
$$

By virtue of Eq. (4), one then has

$$
\begin{aligned}
\langle n\rangle_{\vec{\ell}}= & \left.(1-s) \frac{\partial}{\partial z} F(\vec{\ell}, z)\right|_{z=1-s} \\
& +\left.s(1-s) \frac{\partial}{\partial z}\left\{(1-z)^{-1}(1-F(\vec{\ell}, z))\right\}\right|_{z=1-s} \\
& +\left.s(1-z)^{-1}(1-F(\vec{\ell}, z))\right|_{z=1-s},
\end{aligned}
$$

which can be simplified to obtain

$$
\langle n\rangle_{\vec{\ell}}=\frac{1}{s}(1-F(\vec{\ell}, 1-s)) .
$$

In terms of the generating function of the sojourn probability of an unrestricted walker, one thus has

$$
\langle n\rangle_{\vec{\ell}}=\frac{1}{s}\left(1-\frac{P(\vec{\ell}, 1-s)}{P(\overrightarrow{0}, 1-s)}\right) .
$$

\section{B. Global quantities}

In this subsection we study quantities that are averaged over a uniform set of initial walker-trap configurations (excluding the case where the walker and the trap are initially at the same location). Such quantities are obtained either by fixing the position of the deep trap and summing over the $N-1$ possible positions of the walker that do not overlap with the trap (as done in Ref. [9]), or by fixing the position of the walker and averaging over all possible positions of the deep trap (because of the symmetry of the system, both approaches are equivalent). Here, we choose the second option for convenience.

For the initial-condition averaged probability $A_{T}=$ $\frac{1}{N-1} \sum_{\ell} A_{T}(\vec{\ell})$ of having absorption at the deep trap, Eq. (3) yields

$$
A_{T}=\Gamma(\overrightarrow{0}, 1-s),
$$

where the key quantity

$$
\Gamma(\overrightarrow{0}, z) \equiv \frac{1}{N-1}\left(\frac{1}{(1-z) P(\overrightarrow{0}, z)}-1\right)
$$

has been introduced. When deriving the above result, advantage was taken of the relation, $P(\overrightarrow{0}, z)+P(\vec{\ell}, z)=(1-z)^{-1}$, which follows from the normalization condition $P_{n}(\overrightarrow{0})+$ $\sum_{\ell} P_{n}(\vec{\ell})=1$ for an unrestricted random walk (recall that $\vec{\ell}$ is only allowed to take values different from the origin). The generating function $P(\vec{\ell}, z)$ drops out of the final result for the probability of absorption at the deep trap, and the above quantity is expressible solely in terms of functions of the generating function $P(\overrightarrow{0}, z)$ for the probability of return to the origin in an unrestricted random walk.

For the global walk length $\langle n\rangle=\frac{1}{N-1} \sum_{\vec{\ell}}\langle n\rangle_{\vec{\ell}}$, a quantity that can be used to gauge the efficiency of the absorption process in experiments, we have

$$
\langle n\rangle=\frac{A_{0}}{s}=\frac{1-A_{T}}{s}=\frac{1-\Gamma(\overrightarrow{0}, 1-s)}{s} .
$$

Equation (9) states that the mean walk length can be straightforwardly computed in terms of the probability of absorption at any of the partial traps. The connection between the zeroth and the first order moment of the walk length is not entirely surprising as, in the absence of background absorption, the moments of the distribution are also known to obey a hierarchy of equations [20].

Note also that for increasingly large lattices the probability for the walker to be absorbed at the deep trap is dramatically reduced; if we fix $s, A_{T} \rightarrow 0$ as $N \rightarrow \infty$ and thus $\langle n\rangle \rightarrow s^{-1}$, which is the value of the walk length on a lattice where all sites have the same absorption probability $s$.

The above limiting behavior is not surprising. Indeed, think of the "walker" as a radioactive isotope decaying according to an exponential law $\propto e^{-\lambda t}$ as it diffuses on a homogeneous substrate (absorption is thus ascribed not to the substrate properties, but to radioactive decay). In discretized time, the probability to "die" upon performing a jump is $s=1-e^{-\lambda \Delta t}$ and thus one has $s=\lambda \Delta t$ for small enough $\lambda \Delta t$. On the other hand, $\lambda^{-1}$ is also known to be the mean lifetime of the isotope $\langle t\rangle=\langle n\rangle \Delta t$, from whence $\langle n\rangle=s^{-1}$ follows.

\section{ANALYTIC RESULTS FOR A LINEAR CHAIN}

Though the primary focus of the present work is on higherdimensional template geometries, it is worth noting that exact results for arbitrary values of $s$ and $N$ can be obtained for a onedimensional lattice subject to periodic boundary conditions (topologically, a ring with $N$ lattice sites). In this case, we have Montroll's well-known (exact) result [10(a)]

$$
P(\overrightarrow{0}, z)=\frac{1}{\sqrt{1-z^{2}}} \frac{1+x^{N}}{1-x^{N}},
$$

with $x=z^{-1}\left(1-\sqrt{1-z^{2}}\right)$. After tedious algebra, the use of the above formula for $\langle n\rangle$ yields

$$
\langle n\rangle=\frac{N s+\sqrt{2 s-s^{2}}\left(1-\frac{2}{1+\zeta^{N}}\right)}{s^{2}(N-1)},
$$

with $\zeta=\left(1-\sqrt{2 s-s^{2}}\right) /(1-s)$. Results calculated using the above expression recover exactly the exact numerical results reported in Table 1 of Ref. [8], e.g., for $N=8$ and for $N=20$ with $s=0.1$.

For strong absorption $(s \approx 1)$, the exact result $(10)$ for the walk length can be expanded about $s=1$ as follows:

$$
\langle n\rangle=1+\frac{N-2}{N-1}(1-s)+\frac{2 N-5}{2 N-2}(1-s)^{2}+O\left[(1-s)^{3}\right] .
$$

For weak absorption $(s \approx 0)$ one has

$$
\langle n\rangle=\frac{N(N+1)}{6}-\frac{(N+2)(N+1) N(N-2)}{30} s+O\left(s^{2}\right) .
$$

Note that the first term on the right-hand side corresponds to the $s=0$ case and is a well-known result by Montroll [10]. 
The probability to die at the deep trap can then be obtained from the above results via the exact relation $A_{T}=1-s\langle n\rangle$. For asymptotic results, this expression can be expanded about $s=0$ or $s=1$.

A connection can be established between results obtained assuming no background absorption $(s=0)$ and the problem of computing the mean duration of a game in the classical "ruin problem" (see Feller [21]). It is interesting that this correspondence also holds for the case studied above $(s \neq 0)$. The latter situation corresponds to a modified version of the ruin problem where the gambler (or the bank) has a constant probability $s$ of quitting the game spontaneously after each bet. Thus, the game may not only terminate by breaking the bank or going bankrupt, but also by spontaneous withdrawal.

\section{RESULTS FOR SPECIFIC PLANAR LATTICES}

\section{A. Correspondence between generating function approach and Markov theory}

In order to test the validity of the theoretical expression (9) for the walk length in higher dimensions, we next focus on the particular case of a nearest neighbor, symmetric random walk taking place on a square planar lattice with periodic boundary conditions. For odd lattices, values for the walk length for particular values of the absorption probability $s$ were computed from the solution of a Markov model and given in Table $\mathrm{V}$ of Ref. [9]. From the analysis of these data, a dramatic decrease in the mean walk length with respect to increase in the absorption probability was found.

To recover these results using the generating function approach, we make use of Eq. (9) in conjunction with the generating function for an unrestricted walk on a rectangular planar lattice of $N$ sites, where $N=L_{1} \times L_{2}$ :

$$
P(\overrightarrow{0}, z) \equiv \frac{1}{N} \sum_{k_{1}=0}^{L_{1}-1} \sum_{k_{2}=0}^{L_{2}-1} \frac{1}{1-(z / 2)\left[\cos \left(2 \pi k_{1} / L_{1}\right)+\cos \left(2 \pi k_{2} / L_{2}\right)\right]} .
$$

In what follows, we focus primarily on the case of the square planar lattice $\left(L_{1}=L_{2}=L\right)$ :

$P(\overrightarrow{0}, z) \equiv \frac{1}{N} \sum_{k_{1}=0}^{L-1} \sum_{k_{2}=0}^{L-1} \frac{1}{1-(z / 2)\left[\cos \left(2 \pi k_{1} / L\right)+\cos \left(2 \pi k_{2} / L\right)\right]}$.

Taking Eq. (11) as a starting point, we have obtained rational expressions in terms of $s$-polynomials for the global walk length given in Table I. These expressions are in perfect agreement with the Markov results obtained for odd squareplanar lattices and specific values of $s$; see Table V of Ref. [9]. In Table II, we also provide additional results for the first few even lattices. As the lattice size $N$ becomes larger, the results converge to $s^{-1}$ from below, i.e., $\lim _{N \rightarrow \infty}\langle n\rangle=s^{-1}$. According to the statistical arguments given in [9] and in Sec. II of the present paper, this is the average walk length that would correspond to a walker wandering in a lattice that has been made homogeneous by replacing the deep trap with a partially absorbing site equivalent to the ones surrounding the trap. In the limit of a large lattice, the influence of the deep trap becomes of increasingly less consequence and the results become indistinguishable from the case of a single decay channel.

Finally, in Table III we give for completeness the polynomial expressions for the global absorption probability at the deep trap $A_{T}=1-s\langle n\rangle$ for the first few square lattices as well as for the $3 \times 2$ lattice. Representative results are depicted in Fig. 1, displaying a dramatic decrease of $A_{T}$ with increasing values of $N$, and the correct behavior in the limit $s \rightarrow 0$ and

TABLE I. Analytic results for odd, planar lattices: $\{N, d, \gamma, \Omega: L x L, 2,4,0\}$.

\begin{tabular}{|c|c|}
\hline$N=L \times L$ & $\langle n\rangle$ \\
\hline $3 \times 3$ & $\frac{-9+s}{-1-8 s+s^{2}}$ \\
\hline $5 \times 5$ & $\frac{475+500 s-150 s^{2}-60 s^{3}+3 s^{4}}{15+483 s+474 s^{2}-150 s^{3}-57 s^{4}+3 s^{5}}$ \\
\hline $7 \times 7$ & $\frac{19551+81634 s+48020 s^{2}-45570 s^{3}-12642 s^{4}+7518 s^{5}-84 s^{6}-126 s^{7}+3 s^{8}}{273+20727 s+81480 s^{2}+46008 s^{3}-45246 s^{4}-12114 s^{5}+7392 s^{6}-96 s^{7}-123 s^{8}+3 s^{9}}$ \\
\hline $9 \times 9$ & $\begin{array}{l}\left(3696759+35116335 s+84334608 s^{2}+23183496 s^{3}-66328065 s^{4}-14183505 s^{5}+19050552 s^{6}+697320 s^{7}\right. \\
\left.\quad-1785915 s^{8}+54045 s^{9}+52920 s^{10}-2160 s^{11}-315 s^{12}+5 s^{13}\right) /\left(28305+3994290 s+35753895 s^{2}+83886300 s^{3} a\right. \\
+21978585 s^{4}-65960730 s^{5}-13644985 s^{6}+18868840 s^{7}+641035 s^{8}-1764770 s^{9}+55565 s^{10}+52220 s^{11} \\
\left.\quad-2165 s^{12}-310 s^{13}+5 s^{14}\right)\end{array}$ \\
\hline $11 \times 11$ & $\begin{array}{l}\left(3696427911+63561310043 s+334135569669 s^{2}+593603195977 s^{3}+45005438412 s^{4}-640330869860 s^{5}\right. \\
\quad-163367711980 s^{6}+299687890788 s^{7}+44908224834 s^{8}-72517838822 s^{9}+256667862 s^{10} \\
\quad+7857511310 s^{11}-866550212 s^{12}-281008948 s^{13}+45887556 s^{14}+2545620 s^{15}-622545 s^{16}+6435 s^{17} \\
\left.\quad+1485 s^{18}-15 s^{19}\right) /\left(17607315+4036077210 s+65399016540 s^{2}+336653463630 s^{3}+590537465925 s^{4}\right. \\
\quad+38745850440 s^{5}-638064583200 s^{6}-158901448200 s^{7}+298332459150 s^{8}+43588975980 s^{9}-72034228680 s^{10} \\
\quad+397468260 s^{11}+7785109050 s^{12}-866902680 s^{13}-277793280 s^{14}+45645720 s^{15}-2502255 s^{16} \\
\left.\quad-617910 s^{17}+6540 s^{18}+1470 s^{19}-15 s^{20}\right)\end{array}$ \\
\hline
\end{tabular}


TABLE II. Analytic results for even, planar lattices: $\{N, d, \gamma, \Omega: L x L, 2,4,0\}$.

\begin{tabular}{|c|c|}
\hline$N=L \times L$ & $\langle n\rangle$ \\
\hline $2 \times 2$ & $\frac{10-4 s}{3+6 s-3 s^{2}}$ \\
\hline $4 \times 4$ & $\frac{824-192 s-200 s^{2}+48 s^{3}}{45+780 s-210 s^{2}-180 s^{3}+45 s^{4}}$ \\
\hline $6 \times 6$ & $\frac{60462+104288 s-79662 s^{2}-43500 s^{3}+33458 s^{4}-2088 s^{5}-1458 s^{6}+180 s^{7}}{1225+62300 s+99890 s^{2}-80080 s^{3}-40880 s^{4}+32620 s^{5}-2170 s^{6}-1400 s^{7}+175 s^{8}}$ \\
\hline $8 \times 8$ & $\begin{array}{l}2218528+12816768 s+8978272 s^{2}-19491904 s^{3}-3922880 s^{4}+10393600 s^{5}-2126912 s^{6}-950144 s^{7}+363424 s^{8} \\
\left.\quad-16256 s^{9}-5408 s^{10}+448 s^{11}\right) /\left(22491+2360736 s+12840156 s^{2}+8546580 s^{3}-19379493 s^{4}-3635856 s^{5}\right. \\
\left.\quad+10229184 s^{6}-2140488 s^{7}-922383 s^{8}+357840 s^{9}-16380 s^{10}-5292 s^{11}+441 s^{12}\right)\end{array}$ \\
\hline $10 \times 10$ & $\begin{array}{l}\left(-3256308650-39472636300 s-118558579400 s^{2}-15345099760 s^{3}+207911678528 s^{4}+8701567536 s^{5}\right. \\
\quad-145465668840 s^{6}+31547082160 s^{7}+36021452700 s^{8}-14795114024 s^{9}-2129435608 s^{10}+1929090160 s^{11} \\
\left.\quad-192332240 s^{12}-58410000 s^{13}+12913800 s^{14}-343600 s^{15}-81250 s^{16}+4500 s^{17}\right) /(-19422315-3521449998 s \\
\quad-40246687305 s^{2}-118023217488 s^{3}-13024710468 s^{4}+206747230536 s^{5}+6640541028 s^{6}-143986415760 s^{7} \\
\quad+31922314974 s^{8}+35489278044 s^{9}-14730629958 s^{10}-2068800624 s^{11}+1909640700 s^{12}-192836952 s^{13} \\
\left.\quad-57418812 s^{14}+12784464 s^{15}-344619 s^{16}-80190 s^{17}+4455 s^{18}\right)\end{array}$ \\
\hline $12 \times 12$ & $\begin{array}{l}\left(18249873048+367006931712 s+2138264814456 s^{2}+3356002773168 s^{3}-3267252599712 s^{4}-6945718665088 s^{5}\right. \\
\quad+4748718028640 s^{6}+4100541294144 s^{7}-3821796757808 s^{8}-183567728768 s^{9}+1010399296656 s^{10}-282823395040 s^{11} \\
\quad-32829284128 s^{12}+26441535360 s^{13}-2999143200 s^{14}-363317184 s^{15}+90465624 s^{16}-3203712 s^{17}-285768 s^{18} \\
\left.\quad+14256 s^{19}\right) /\left(70855785+19866593604 s+376962592578 s^{2}+2150333360604 s^{3}+3321525822329 s^{4}-3306677438064 s^{5}\right. \\
-6877690997320 s^{6}+4767003349680 s^{7}+4043388448242 s^{8}-3807307521160 s^{9}-169387729940 s^{10} \\
+1001462998536 s^{11}-282004748454 s^{12}-32159809968 s^{13}+26240231160 s^{14}-2993452176 s^{15} \\
\left.\quad-358221435 s^{16}+89775972 s^{17}-3194334 s^{18}-283140 s^{19}+14157 s^{20}\right)\end{array}$ \\
\hline
\end{tabular}

$s \rightarrow 1$. The analytic results for $\langle n\rangle$ inferred from the $A_{T}$ values given in Table III can be compared with those reported earlier for the global absorption probability for random walks on the surface of five polyhedra, the Platonic solids [22]; see Table IV.

\section{B. Large-s limit (minimal degradation)}

The analytical results depicted in Tables II and III are exact and valid for small lattices of increasing size. In the limit of $s$ close to 1 and of $s$ close to 0 , rather general results can be obtained. For a catalyst, the first situation would correspond to weak poisoning, whereas the second limit would correspond to strong poisoning (see Sec. IV C.).

In this subsection we discuss the limit of weak poisoning, which corresponds to $z=1-s \approx 0^{+}$. In this case, $P(\overrightarrow{0}, z)$ can be expanded as follows:

$$
P(\overrightarrow{0}, z)=1+\frac{1}{4} z^{2}+O\left(z^{4}\right), \quad L \geqslant 3 .
$$

This expansion follows by using the expansion $(1-C z)^{-1}=1+C z+C^{2} z^{2}+O\left(z^{3}\right)$ in the definition of

TABLE III. Absorption probabilities at the deep trap for planar lattices subject to periodic boundary conditions: $\{N, d, \gamma, \Omega: L x L, 2,4,0\}$.

\begin{tabular}{lc}
\hline \hline$L_{1} \times L_{2}$ & $A_{T}$ \\
\hline $2 \times 2$ & $\frac{3-4 s+s^{2}}{3+6 s-3 s^{2}}$ \\
$3 \times 2$ & $\frac{-35+37 s-s^{2}-s^{3}}{5\left(-7-33 s+7 s^{2}+s^{3}\right)}$ \\
$3 \times 3$ & $\frac{-1+s}{-1-8 s+s^{2}}$ \\
$4 \times 4$ & $\frac{45-44 s-18 s^{2}+20 s^{3}-3 s^{4}}{45+780 s-210 s^{2}-180 s^{3}+45 s^{4}}$ \\
$5 \times 5$ & $\frac{15+8 s-26 s^{2}+3 s^{4}}{15+483 s+474 s^{2}-150 s^{3}-57 s^{4}+3 s^{5}}$ \\
$6 \times 6$ & $\frac{1225+1838 s-4398 s^{2}-418 s^{3}+2620 s^{4}-838 s^{5}-82 s^{6}+58 s^{7}-5 s^{8}}{1225+62300 s+99890 s^{2}-80080 s^{3}-40880 s^{4}+32620 s^{5}-2170 s^{6}-1400 s^{7}+175 s^{8}}$ \\
\hline
\end{tabular}




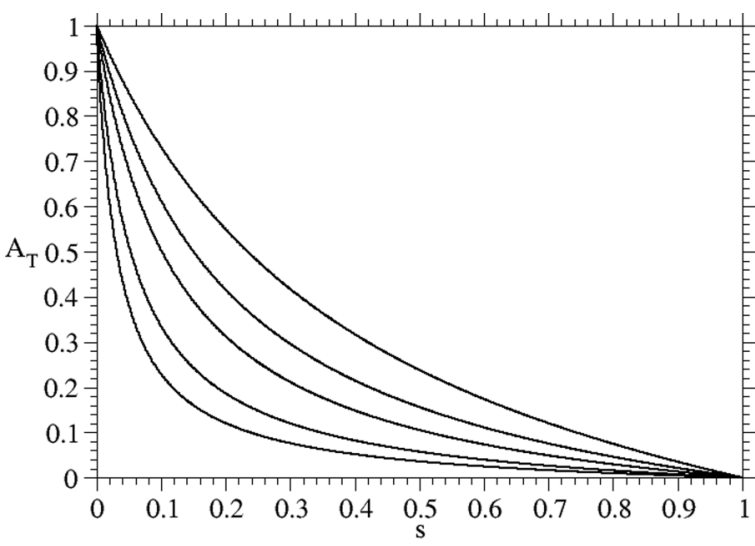

FIG. 1. Initial-condition averaged probability $A_{T}$ of absorption at the deep trap as a function of the absorption probability $s$ of the partially absorbing sites. The different curves correspond, from top to bottom, to $N=4(2 \times 2$ lattice $), 6(3 \times 2$ lattice $), 9(3 \times 3$ lattice $)$, $16(4 \times 4$ lattice $)$, and $25(5 \times 5$ lattice $)$.

$P(\overrightarrow{0}, z)$ [Eq. (11)], and subsequently using the relation

$$
\sum_{k_{1}=0}^{L-1} \sum_{k_{2}=0}^{L-1}\left[\cos \left(2 \pi k_{1} / L\right)+\cos \left(2 \pi k_{2} / L\right)\right]^{2}=L^{2}
$$

to compute the coefficient of the $z^{2}$ term. Inserting the expansion (12) into Eq. (7) and expanding once again the resulting expression in powers of $1-s$ one finds

$$
\begin{aligned}
A_{T}= & \frac{1}{N-1}(1-s)+\frac{3}{4(N-1)}(1-s)^{2} \\
& +O\left[(1-s)^{3}\right], \quad L \geqslant 3,
\end{aligned}
$$

and hence

$$
\begin{aligned}
\langle n\rangle= & \frac{1-A_{T}}{s}=1+\frac{N-2}{N-1}(1-s) \\
& +\frac{4(N-1)-7}{4(N-1)}(1-s)^{2}+O\left[(1-s)^{3}\right], \quad L \geqslant 3 .
\end{aligned}
$$

The case of the $2 \times 2$ lattice must be treated separately, since in this case $\sum_{k_{1}=0}^{1} \sum_{k_{2}=0}^{1}\left[\cos \left(\pi k_{1}\right)+\cos \left(\pi k_{2}\right)\right]^{2}=8 \neq$ 4. One thus has $P(\overrightarrow{0}, z)=1+(1 / 2) z^{2}+O\left(z^{4}\right)$. Accordingly, a similar calculation as the one outlined above gives

$$
A_{T}=\frac{1}{3}(1-s)+\frac{1}{6}(1-s)^{2}+O\left[(1-s)^{3}\right], \quad(L=2),
$$

and

$$
\langle n\rangle=1+\frac{2(1-s)}{3}+\frac{1}{2}(1-s)^{2}+O\left[(1-s)^{3}\right], \quad(L=2) .
$$

\section{Small-s limit (significant degradation)}

In order to obtain results corresponding to the limit of strong poisoning, we first split the generating function $P(\overrightarrow{0}, z)$ in a standard fashion, namely, as a sum of a part which is singular as $z \rightarrow 1$ and a nonsingular part $\Phi(\overrightarrow{0}, z)$,

$$
P(\overrightarrow{0}, z)=\frac{1}{N(1-z)}+\Phi(\overrightarrow{0}, z) .
$$

We now take advantage of the well-known form of the asymptotic expansion for $P(\overrightarrow{0}, z)$ (see, e.g., Ref. [16]):

$$
P(\overrightarrow{0}, z)=\frac{1}{N(1-z)}+\Phi-\Phi^{\prime}(1-z)+\cdots z \rightarrow 1^{-},
$$

where the lattice size expansion of the coefficients $\Phi \equiv \Phi(\overrightarrow{0}, 1)$ and $\left.\Phi^{\prime} \equiv \Phi^{\prime}(\overrightarrow{0}, 1) \equiv \partial_{z} \Phi(\overrightarrow{0}, z)\right|_{z=1}$ reads as follows:

$$
\begin{aligned}
& \Phi=a_{1} \ln N+a_{2}+a_{3} N^{-1}+a_{4} N^{-2}+\cdots, \\
& \Phi^{\prime}=b_{1}+b_{2} \ln N+b_{3}+b_{4} N^{-1}+b_{5} N^{-2}+\cdots
\end{aligned}
$$

with

$$
\begin{aligned}
& a_{1}=\pi^{-1}, \quad b_{1}=0.061871145451 \ldots, \\
& a_{2}=0.195062532 \ldots, \quad b_{2}=-(2 \pi)^{-1}, \\
& a_{3}=-0.116964779 \ldots, \quad b_{3}=-0.1347623119 \ldots, \\
& a_{4}=0.484065704 \ldots, \quad b_{4}=0.2005850758 \ldots, \\
& b_{5}=0.4283683639 \ldots
\end{aligned}
$$

Inserting the asymptotic expansion (13) into Eq. (7) and using the expansion

$$
\begin{aligned}
& \left(a+b x-c x^{2}\right)^{-1} \\
& \quad=a^{-1}-b a^{-1} x+a^{-3}\left(b^{2}+a c\right) x^{2}+O\left(x^{3}\right),
\end{aligned}
$$

it follows that

$$
A_{T}=1-\Phi \frac{N^{2}}{N-1} s+\frac{N^{3}}{N-1}\left[\Phi^{2}+\frac{\Phi^{\prime}}{N}\right] s^{2}+O\left(s^{3}\right) .
$$

If one retains all of the above terms in the lattice size expansions of $\Phi$ and $\Phi^{\prime}$, this formula yields very good results even for small lattices. For instance, in the $2 \times 2$ case the formula gives $A_{T}=1-3.39918 s+8.5001 s^{2}$, whereas the Taylor expansion of the exact result about $s=0$ gives $A_{T}=1-3.3333 s+8 s^{2}$. For the $3 \times 3$ case, the agreement is already very good; one finds $A_{T}=1-8.9853 s+72.7776 s^{2}$ from the above formula and $A_{T}=1-9 s+73 s^{2}$ from the Taylor expansion. Note, however, that higher order terms in the expansion representing further nonlinear corrections are important. For instance, even for values as small as $s=0.05$, the $s^{3}$ term in the Taylor expansion $\left(593 s^{3}\right)$ is about $40.6 \%$ of the $s^{2}$ term $\left(73 s^{2}\right)$.

TABLE IV. (From Ref. [22].) Mean walk length for polyhedral lattice surfaces: $\{N, d, \gamma, \Omega: N, 2,4,2\}$.

\begin{tabular}{lc}
\hline \hline$N$ & $\langle n\rangle$ \\
\hline 4 & $3 /(1+2 s)$ \\
6 & $(2 / 5)(-13+3 s) /\left(-1-4 s+s^{2}\right)$ \\
8 & $(1 / 7)\left(116-52 s-s^{2}\right) /\left(2+14 s-7 s^{2}\right)$ \\
12 & $(5 / 11)\left(56+4 s-5 s^{2}\right) /\left(2+24 s+s^{2}-2 s^{3}\right)$ \\
20 & $(1 / 19)\left(1096+1388 s-975 s^{2}-10 s^{3}+40 s^{4}\right) /\left(2+60 s+67 s^{2}-50 s^{3}+2 s^{5}\right)$ \\
\hline \hline
\end{tabular}


As for the walk length, from the relation $\langle n\rangle=s^{-1}\left(1-A_{T}\right)$ one finds

$$
\langle n\rangle=\Phi \frac{N^{2}}{N-1}-\frac{N^{3}}{N-1}\left[\Phi^{2}+\frac{\Phi^{\prime}}{N}\right] s+O\left(s^{2}\right) .
$$

In the limit of a very large lattice $(N \rightarrow \infty)$ one obtains the asymptotic expressions

$$
A_{T}=1-\pi^{-1}(N \ln N) s+\left(b_{1} N^{2}+\pi^{-2} N^{2} \ln ^{2} N\right) s^{2}+O\left(s^{3}\right)
$$

and

$$
\langle n\rangle=\pi^{-1} N \ln N-\left(b_{1} N^{2}+\pi^{-2} N^{2} \ln ^{2} N\right) s+O\left(s^{2}\right),
$$

where the first term on the right-hand side corresponds to the well-known Montroll result [10(c)], and the second term is the leading order correction for background absorption.

\section{EXACT RESULTS FOR PLANAR LATTICES AND POLYHEDRAL SURFACES}

Displayed in Tables V and VI are representative numerical results on catalyst degradation obtained from a Markov theory approach similar to the one used in Refs. [8,9]. The starting point of this method is the linear set of equations

$$
\langle n\rangle_{\vec{\ell}}=1+(1-s) \sum_{\overrightarrow{\ell^{\prime}}} p_{\vec{\ell} \rightarrow \vec{\ell}^{\prime}}\langle n\rangle_{\vec{\ell}^{\prime}}
$$

for the site specific walk lengths, where $p_{\vec{\ell} \rightarrow \vec{\ell}^{\prime}}$ represent the transition probabilities from a specific nontrapping site $\vec{\ell}$ to nearest-neighbor sites $\vec{\ell}^{\prime}$ (in the above equations $\langle n\rangle_{\vec{\ell}^{\prime}}$ is taken to be zero if it corresponds to the location of a deep trap). The transition probabilities obey the normalization condition, i.e., $\sum_{\vec{\ell}^{\prime}} p_{\vec{\ell} \rightarrow \vec{\ell}^{\prime}}=1$. Once the site specific walk lengths have been found, the global walk length follows as $\langle n\rangle=\frac{1}{N-1} \sum_{\vec{\ell}}\langle n\rangle_{\vec{\ell}}$. In most cases, the number of equations in the above linear system can be reduced by lumping symmetry-equivalent sites into a single state of the underlying Markov chain. Last but not least, we note that Eq. (14) can be derived from the master equation for the sojourn probabilities $P_{n}(\vec{\ell})$ and the associated generating function $P(\vec{\ell}, z)$ of an unrestricted random walk by standard techniques [cf. Eq. (6)].

Companion results are presented in Table $\mathrm{V}$ for the walk length (whose inverse is a measure of the reaction efficiency of the substrate) for a template having $N=6,54,96,150$ sites modelled as a planar surface (lattice subject to periodic boundary conditions) vs a polyhedral surface, both surfaces having a single, fully active catalytic site and $N-1$ surrounding satellite sites of specified activity, $0 \leqslant s \leqslant 1$. Numerical results for planar lattices subject to periodic boundary conditions can be obtained either by using the generating function approach or Markov theory. Values for polyhedral surfaces are (only) obtained using Markov theory.

To contrast the results obtained above for the case of a single, fully catalytic site (deep trap with absorption probability $T=1$ ) and $N-1$ partially absorbing satellite sites $(s \neq 0)$, we present in Table $\mathrm{V}$ values of the walk length for two catalytically active sites (both with absorption probability $T=T^{*}=1$ ), maximally separated, on the designated planar or polyhedral template, the catalytic activity of the remaining $N-2$ satellite sites totally suppressed $(s=0)$.
Focusing on the walk length, Table VI gives a more detailed accounting of the differences between the planar surface and the polyhedral surface for the particular case, $s=0$. A centrally located active site on a polyhedral face is designated " 1 ," a nearest-neighbor site to site 1 is designated " 2 ," etc.

Conclusions and trends drawn from the results displayed in Tables V and VI will be presented in the following section.

\section{DISCUSSION}

We have developed a lattice-statistical approach to study systems with competing reaction channels, focusing on the problem of catalyst deactivation. We have taken advantage of two complementary theoretical strategies, the first based on the use of generating functions, and the second on the theory of finite Markov processes. Each of these two approaches has particular advantages, and we shall draw on results obtained using both approaches to discuss the catalysis problem addressed here.

One of the first applications of the theory of generating functions was to a problem of fundamental importance in biophysics. Montroll [10(c)] considered the random motion of an excitation on the chlorophyll antenna network (modelled as a lattice) before being localized at a preferred site, subsequently triggering the photosynthetic process. Later work on this model explored the consequences of competing reaction centers, and their effect on the efficiency of the process [9]. In the latter study, a dramatic dependence of the mean walk length $\langle n\rangle$ on satellite-site trapping probability $s$ emerged, namely

$$
\lim _{N \rightarrow \infty}\langle n\rangle=s^{-1} .
$$

The catalyst deactivation problem considered here can be regarded as the "mirror image" of the chlorophyll antenna problem. In the latter, small changes in $s$ in the vicinity of $s \approx 0$ compromise dramatically the light-energy conversion efficiency and the limiting value (above) is approached asymptotically. In the catalyst degradation problem, the scenario is exactly the reverse. For an "ideal" catalyst, all sites $N$ lead to conversion, and their degradation (values of $s$ decreasing from $s=1$ ) gradually changes the efficiency of the process, with complete "poisoning" in the regime $s \rightarrow 0$. Thus, the optimal regime for photosynthetic activity is in the vicinity $s \rightarrow 0$, whereas the optimal regime for catalytic activity is in the range $s \rightarrow 1$.

Focusing specifically on catalyst degradation, the results presented in Table $\mathrm{V}$ for catalysts defined by small domains (relatively few active sites $N$ ) show that $\langle n\rangle$ is less sensitive to poisoning of closed polyhedral surfaces mimicking catalyst pellets than on planar surfaces, whereas exactly the opposite situation pertains for larger system sizes. One possible conclusion that can be drawn from these data is that nanoparticles are likely to be more immune to poisoning than nanosurfaces, with the situation reversed above a critical domain size.

If one associates the mean walk length $\langle n\rangle$ with a mean time $\langle\tau\rangle$ for conversion and the inverse $\langle\tau\rangle^{-1}$ to an estimate of the rate constant, departures from the $1 / s$ dependence of the inverse rate constant (associated with a nonlinear dependence of the rate constant on concentration, see below) have been 
TABLE V. Reaction between a diffusing atom or molecule (the walker) and competing reaction centers for selected geometries. The probability of irreversible absorption at the target molecule is specified by $T=1$ (deep trap), at a competing reaction site by $T^{*}$, and at satellite sites by $0 \leqslant s \leqslant 1$.

\begin{tabular}{|c|c|c|c|}
\hline \multicolumn{2}{|l|}{$N, d, \gamma, \Omega: 6,2,4,0$} & \multicolumn{2}{|l|}{$N, d, \gamma, \Omega: 6,2,4,2$} \\
\hline Abs. prob. $T, T^{*}, s$ & $\langle n\rangle$ & Abs. prob. $T, T^{*}, s$ & $\langle n\rangle$ \\
\hline 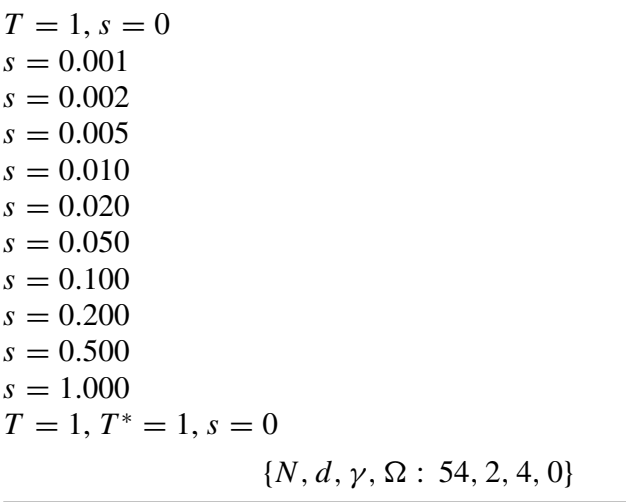 & $\begin{array}{l}5.771 \\
5.743 \\
5.715 \\
5.633 \\
5.502 \\
5.257 \\
4.638 \\
3.878 \\
2.923 \\
1.688 \\
1 \\
1.362\end{array}$ & $\begin{array}{c}T=1, s=0 \\
T=1, T^{*}=1, s=0 \\
\quad\{N, d, \gamma, \Omega: 54,2,4,2\}\end{array}$ & $\begin{array}{c}5.2 \\
5.178 \\
5.156 \\
5.092 \\
4.989 \\
4.794 \\
4.292 \\
3.655 \\
2.818 \\
1.672 \\
1 \\
1.333\end{array}$ \\
\hline $\begin{array}{l}\text { Plane } 9 \times 6 \text { torus } \\
T=1, s=0 \\
s=0.001 \\
s=0.002 \\
s=0.005 \\
s=0.010 \\
s=0.020 \\
s=0.050 \\
s=0.100 \\
s=0.200 \\
s=0.500 \\
\mathrm{~s}=1.000 \\
T=1, T^{*}=1, s=0 \\
\quad \quad\{N, d, \gamma, \Omega: 96,2,4,0\}\end{array}$ & $\begin{array}{c}82.480 \\
76.147 \\
70.719 \\
58.263 \\
45.048 \\
31.003 \\
16.041 \\
8.907 \\
4.724 \\
1.967 \\
1 \\
34.634\end{array}$ & $\begin{array}{c}T=1, s=0 \\
T=1, T^{*}=1, s=0 \\
\quad\{N, d, \gamma, \Omega: 96,2,4,2\}\end{array}$ & $\begin{array}{c}84.544 \\
77.890 \\
72.190 \\
59.219 \\
45.582 \\
31.224 \\
16.082 \\
8.914 \\
4.724 \\
1.967 \\
1 \\
34.520\end{array}$ \\
\hline $\begin{array}{l}\text { Plane } 12 \times 8 \text { torus } \\
T=1, s=0 \\
s=0.001 \\
s=0.002 \\
s=0.005 \\
s=0.010 \\
s=0.020 \\
s=0.050 \\
s=0.100 \\
s=0.200 \\
s=0.500 \\
s=1.000 \\
T=1, T^{*}=1, s=0 \\
\qquad\{N, d, \gamma, \Omega: 150,2,4,0\}\end{array}$ & $\begin{array}{c}163.233 \\
140.075 \\
122.677 \\
89.388 \\
61.572 \\
37.977 \\
17.699 \\
9.382 \\
4.846 \\
1.982 \\
1 \\
70.094\end{array}$ & $\begin{array}{l}T=1, s=0 \\
T=1, T^{*}=1, s=0 \\
\quad\{N, d, \gamma, \Omega: 150,2,4,2\}\end{array}$ & $\begin{array}{c}168.620 \\
143.941 \\
125.570 \\
90.823 \\
62.184 \\
38.169 \\
17.723 \\
9.385 \\
4.846 \\
1.982 \\
1 \\
71.158\end{array}$ \\
\hline $\begin{array}{l}\text { Plane } 15 \times 10 \text { torus } \\
T=1, s=0 \\
s=0.001 \\
s=0.002 \\
s=0.005 \\
s=0.010 \\
s=0.020 \\
s=0.050 \\
s=0.100 \\
s=0.200 \\
s=0.500 \\
s=1.000 \\
T=1, T^{*}=1, s=0\end{array}$ & $\begin{array}{c}275.572 \\
215.392 \\
176.799 \\
115.021 \\
72.725 \\
41.943 \\
18.515 \\
9.605 \\
4.901 \\
1.988 \\
1 \\
119.291\end{array}$ & $T=1, T^{*}=1, s=0$ & $\begin{array}{c}282.710 \\
219.571 \\
179.504 \\
116.045 \\
73.070 \\
42.026 \\
18.522 \\
9.606 \\
4.901 \\
1.988 \\
1 \\
119.448\end{array}$ \\
\hline
\end{tabular}


TABLE VI. Reaction between a diffusing atom or molecule (walker) and a stationary target molecule (deep trap) for selected geometries $\{N, d, \gamma, \Omega\}: N$ is the number of surface sites, $d$ is the Euclidean dimension, $\gamma$ is the connectivity of the reaction space, and $\Omega$ is the Euler characteristic of the surface.

\begin{tabular}{|c|c|c|c|c|}
\hline$(N, d, \gamma ; \Omega)$ & Surface & Boundary conditions & Reaction center & Walk length $(\langle n\rangle)$ \\
\hline$\{6,2,4,0\}$ & plane & $3 \times 2$ torus & any site & 5.771428571 \\
\hline$\{6,2,4,2\}$ & octahedron & closed & any site & 5.2 \\
\hline \multirow[t]{2}{*}{$54,2,4,0$} & plane & $9 \times 6$ torus & any site & 82.48027996 \\
\hline & & $18 \times 3$ torus & any site & 141.7143833 \\
\hline \multirow[t]{3}{*}{$54,2,4,2$} & polyhedron & closed & any site 1 & 84.54384823 \\
\hline & & & any site 2 & 86.20603538 \\
\hline & & & any site 3 & 90.34311180 \\
\hline \multirow[t]{2}{*}{$96,2,4,0$} & plane & $12 \times 8$ torus & any site & 163.2332366 \\
\hline & & $32 \times 3$ torus & any site & 401.0412934 \\
\hline \multirow[t]{3}{*}{$96,2,4,2$} & polyhedron & closed & any site 1 & 168.6198973 \\
\hline & & & any site 2 & 172.1422298 \\
\hline & & & any site 3 & 182.5297641 \\
\hline \multirow[t]{2}{*}{$150,2,4,0$} & plane & $15 \times 10$ torus & any site & 275.5720626 \\
\hline & & $32 \times 3$ torus & any site & 926.4635800 \\
\hline \multirow[t]{5}{*}{$150,2,4,2$} & polyhedron & closed & any site 1 & 282.7097284 \\
\hline & & & any site 2 & 284.5828348 \\
\hline & & & any site 3 & 287.5025078 \\
\hline & & & any site 4 & 288.0941334 \\
\hline & & & any site 5 & 312.3516256 \\
\hline
\end{tabular}

seen in numerous experimental studies. For example, in a classic study of the $\mathrm{CO}$ poisoning of para- $\mathrm{H}_{2}$ conversion over a $\mathrm{Pt}$ foil, one finds a gradual change in conversion rate between $1 \times 10^{14}$ and $5 \times 10^{14}$ molecules of $\mathrm{CO}$ per $\mathrm{cm}^{-2}$ with a more pronounced change as the concentration approaches $10^{15}$ molecules $/ \mathrm{cm}^{2}$ [23]. Although poisoning on a $\mathrm{Pt}$ foil and poisoning on (an imagined) Pt pellet would both exhibit an eventual $1 / s$ dependence on CO concentration, the approach to this $1 / s$ dependence is analytically different for the two geometries. The simplest case for which results can be compared directly is for a template of $N=6$ active sites. For a nanosurface of $N=3 \times 2=6$ sites, the behavior is (cf. Table III)

$$
\langle n\rangle=(2 / 5)\left(-101+18 s+3 s^{2}\right) /\left(-7-33 s+7 s^{2}+s^{3}\right)
$$

(this result can be derived using either the generating-function approach or Markov theory). In contrast, for a nanopellet (Table IV), the dependence is [22]

$$
\langle n\rangle=(2 / 5)(-13+3 s) /\left(-1-4 s+s^{2}\right) .
$$

The numerical consequences of opting for one or the other of these topologies for domain sizes $N>6$ are documented in Table $\mathrm{V}$ for various settings of $s$. As $s$ decreases, the walk length is seen to increase faster on a planar surface than on a polyhedral surface. Already for the next domain size reported in Table $\mathrm{V}$, a support having $N=54$ active sites, the behavior is the opposite, i.e., poisoning of a catalyst surface is of somewhat less consequence than for a catalyst pellet. Such behavior is also observed for $N=96$ and 150 .

Two topologically distinct models of a catalyst template have been considered in this study, the first a planar surface (a discrete lattice subject to periodic boundary conditions with Euler characteristic $\Omega=0$ ) and the second a pellet (a cube with domains of square-planar symmetry on each of the six faces with Euler characteristic $\Omega=2$ ). Since catalysts are neither "perfect" planar surfaces nor "perfect" cubes, it is important to address the relevance of our results to "real" catalyst geometries.

We begin by noting that only passive diffusion is considered in this study, that is, we assume that a species migrating on a template having a specified array of reaction sites is not subject to any distance-dependent, long-range forces. In this case, for a given specification of $\Omega$, the surface being modelled can be diffeomorphically distorted into any topological shape provided no "cuts" or other disjoint seams are introduced. All that matters is the Euclidean dimension of the lattice (here, $d=2$ ), the total number $N$ of lattice sites defining the domain, and their connectivity $\gamma$ (here all sites are of uniform coordination, $\gamma=4$ ). Topologically then, an "ideal" planar or polyhedral surface can be deformed into any geometry having the same Euler characteristic $\Omega$ (however "irregular") and trends deduced from results calculated for a given setting of $[N, d, \gamma]$ remain valid.

The results reported in Table $\mathrm{V}$ have relevance to the problem of chip design. Presented there are values of $\langle n\rangle$ calculated for one fully active catalytic site, with $N-1$ satellite sites assigned a range of values, $s \neq 0$. These can be compared with values of $\langle n\rangle$ for the case where the full catalytic activity of two sites is maintained (both are "deep traps") but the catalytic activity of the remaining $N-2$ satellite sites totally suppressed $(s=0)$. Spatially, the two active sites are specified to be maximally separated on the template. We find that there is a crossover value of the satellite absorption probability $s$ for which $\langle n\rangle$ values calculated for both chip fabrications are in correspondence. This crossover value of $s$ shifts to smaller values as the lattice size $N$ increases. Thus, the catalytic effectiveness of a few (here two) fully active sites on a small chip can match the catalytic activity of a 
companion chip with a single active site and $N-1$ partially absorbing sites. It is noteworthy that this correspondence can be maintained for larger chip sizes only if satellite reactivity is further suppressed. We suggest that this feature may be important in the design of nanosystem sensors.

The consequences of assigning different positions of a catalytically active site on the surface of a polyhedron of $N$ sites are explored in Table VI. Numerical differences in values of $\langle n\rangle$ can be significant. By contrast, all sites on a planar lattice for which translational invariance holds are equivalent, so the calculated walk length to any assigned site remains invariant. However, for planar templates of arbitrary shape translational invariance may be lost, in which case the Euler characteristic reverts to $\Omega=2$, all sites are not equivalent, and, like an arbitrary (non-Platonic) polyhedral surface, the calculated mean walk length will depend on the position of the catalytically active site(s) with respect to the boundary of the domain.

To summarize the principal results of our study, we have shown that the presence of competing reaction channels can have a dramatic effect on the efficiency of the underlying diffusion-reaction process. We have quantified the role of system geometry (specified by the domain size $N$, dimensionality $d$, connectivity $\gamma$, and Euler characteristic $\Omega$ ) and site-specific reaction rates (as gauged by the absorption probability $s$ ). While our lattice model is admittedly a schematic characterization of a real catalyst system, it has the virtue of capturing essential behavior, thus contributing to an understanding of the interplay between reactivity and surface geometry.

For a catalyst with all sites being identical, the $1 / s$ behavior of $\langle n\rangle$ has the following implication. If the site degradation probability $p=1-s$ is assumed to depend linearly on the concentration $c$ of the poisoning agent, then the efficiency of catalytic conversion as measured by the normalized rate $1 /\langle n\rangle$ will follow a linear law in $c$. However, for a small enough catalyst it is enough that a single site remains unaffected by poisoning to see how a more complicated (nonlinear) $c$ dependence can arise.

Nonlinear dependencies of the efficiency of catalytic conversion on $c$ are well documented in the literature (see Ref. [23]). For example, as is often the case for the protonic sites of acid zeolites [24], selective poisoning due to differences in the affinity of individual sites with respect to the chemisorbed poison species can occur. Even in a scenario where only a single site remains fully active (considered in the present study) the calculations reveal that the observed deviations from linear behavior in $c$ are very sensitive to the topological details of the surface. Such deviations have been characterized in detail by analysis of some representative examples.
The importance of providing a detailed characterization of geometric effects in heterogeneous catalysis and their relevance to industrial applications is self-evident. A straightforward generalization of our model would allow more realistic scenarios involving more than one fully active site or even a random collection there of (in Ref. [25] specific cases have been addressed with Markov methods, but a more comprehensive understanding of the interplay between geometry and reaction kinetics is needed). Certain cases with two fully active sites have already been studied here, and we note that our results for odd square planar lattices may be relevant for systems with more than one fully active site. Indeed, the problem of computing the walk length distribution for an odd square planar $N$-site lattice with periodic boundary conditions is mathematically equivalent to computing the same distribution for a lattice composed of an arbitrary number of juxtaposed lattices identical with the original one. Since the original $N$-site lattice is replicated several times, one ends up with more than one trap in the final lattice. Large (small) values of the sublattice size $N$ would correspond to a dilute (high) concentration of reaction centers (deep traps).

The present study is in the same genre as other lattice models reported in the literature which capture salient features of catalysis, for example the now-classical Ziff-Gulari-Barshad model for the oxidation of carbon monoxide to carbon dioxide on a catalyst surface [26]. That said, to provide a more realistic description of real systems, the present work can be extended in several directions. From the point of view of catalyst deactivation, the effect of introducing more complex inhomogeneous landscapes is of interest as is introducing time-dependent absorption probabilities and considering down-range forces between the diffusing reactant and an ensemble of reaction centers. We anticipate that interesting kinetic effects will arise when the assumption of reaction irreversibility is relaxed or mobility of reaction centers is introduced. Progress along these lines will be reported in the near future.

\section{ACKNOWLEDGMENTS}

E.A. gratefully acknowledges financial support from the Spanish Government through Grant No. FIS2013-42840-P (partially funded by FEDER funds) as well as additional financial support from Junta de Extremadura through Grant No. GR10158. Part of this study was carried out while E.A. was a visiting professor at DePaul University, and while J.J.K. was in residence at the Beckman Institute. The latter author thanks H. B. Gray for his support and generous hospitality.
[1] C. H. Bartholomew, Appl. Catal., A 212, 17 (2001).

[2] R. Imbihl and G. Ertl, Chem. Rev. 95, 697 (1995); G. Nicolis and I. Prigogine, Self-Organization in Nonequilibrium Systems (John Wiley \& Sons, New York, 1977).

[3] A. Wheeler and A. J. Robell, J. Catal. 13, 299 (1969).
[4] J. Markos, A. Brunovska, and J. Ilavsky, Chem. Papers 41, 375 (1987).

[5] C. A. Voigt and R. M. Ziff, J. Chem. Phys. 107, 7397 (1997).

[6] E. V. Albano and J. Marro, J. Chem. Phys. 113, 10279 (2000).

[7] J. P. Bodanese and M. Samos, Braz. J. Phys. 34, 425 (2004).

[8] C. A. Walsh and J. J. Kozak, Phys. Rev. Lett. 47, 1500 (1981). 
[9] C. A. Walsh and J. J. Kozak, Phys. Rev. B 26, 4166 (1982).

[10] (a) E. W. Montroll, in Proceedings of the Symposium on Applied Mathematics (American Mathematical Society, Providence, R.I., 1964), Vol. 16, p. 193; (b) E. W. Montroll and G. H. Weiss, J. Math. Phys. 6, 167 (1965); (c) E. W. Montroll, ibid. 10, 753 (1969).

[11] S. B. Yuste, E. Abad, and K. Lindenberg, Phys. Rev. Lett. 110, 220603 (2013); Arrival atatistics and exploration properties of mortal walkers, in First Passage Phenomena and Their Applications (World Scientific, Singapore, 2014).

[12] H. B. Rosenstock, J. Soc. Ind. Appl. Math. 9, 169 (1961).

[13] H. B. Rosenstock, J. Math. Phys. 11, 487 (1970).

[14] N. B. Levinson, J. Soc. Ind. Appl. Math. 10, 442 (1962).

[15] M. Rudemo, SIAM J. Appl. Math. 14, 1293 (1966).

[16] W. T. F. den Hollander and P. W. Kasteleyn, Physica A 112, 523 (1982).

[17] J. W. Evans and R. S. Nord, Phys. Rev. A 32, 2926 (1985).

[18] G. H. Weiss, J. Stat. Phys. 44, 933 (1986).
[19] G. H. Weiss, Aspects and Applications of the Random Walk (North-Holland, Amsterdam, 1994).

[20] See, e.g., O. Bénichou, C. Chevalier, J. Klafter, B. Meyer, and R. Voituriez, Nat. Chem. 2, 472 (2010); for the continuum analog see S. Redner, A guide to first-passage processes (Cambridge University Press, Cambridge, England, 2001).

[21] W. Feller, An Introduction to Probability Theory, Vol. 1 (John Wiley \& Sons, New York, 1968).

[22] P. A. Politowicz, R. A. Garza-López, D. E. Hurtubise, and J. J. Kozak, J. Phys. Chem. 93, 3728 (1989).

[23] V. J. Volter and M. Hermann, Z. Anorg. All. Chem. 405, 315 (1974).

[24] M. Guisnet, Poisoning of zeolite catalysts, in Deactivation and Regeneration of Zeolite Catalysts, edited by M. Guisnet and F. Ramoa Ribeiro (World Scientific, Singapore, 2011).

[25] P. A. Politowicz and J. J. Kozak, Langmuir 1, 429 (1985).

[26] R. M. Ziff, E. Gulari, and Y. Barshad, Phys. Rev. Lett. 56, 2553 (1986). 